\title{
ENUMERATION OF LATTICE PATHS AND GENERATING FUNCTIONS FOR SKEW PLANE PARTITIONS
}

Christian

Krattenthaler

\begin{abstract}
n-dimensional lattice paths not touching the hyperplanes $x_{1}-x_{t+1}=-1, \dot{F}=1,2, \ldots, n$, are counted by four different otatistics, one of which is MacMahon's major index. By a reflection-like proof, heavily relying on Zeilberger's (Discrete Math. 44(1983), 325-326) solution of the n-candidate ballot problem, determinantal expressions are obtained. As corollaries the generating functions for skew plane partitions, column-strict skew plane partitions, reverse skew plane plane partitions and column-strict reverse skew plane partitions, respectively, are evaluated, thus establishing partly new results, partly new proofs for known theorems in the theory of plane partitions.
\end{abstract}

1. INTRODUCTION. Consider n-dimensional lattice paths, consisting of positive unit steps, from 0 to $\lambda=\left(\lambda_{1}, \lambda_{2}, \ldots, \lambda_{n}\right)$ such that all the points $\left(x_{1}, x_{2}, \ldots, x_{n}\right)$ of those paths satisfy $x_{1} \geqslant x_{2} \cdots \cdots x_{n}$. The problem of counting the number of all those lattice paths is equivalent to the n-candidate ballot problem and has attained several solutions (see [12] for references). In this paper, more generally, we encounter the problem of counting all lattice paths from $\mu=\left(\mu_{3}, \mu_{2}, \ldots, \mu_{n}\right)$ to $\lambda_{\text {, each }}$ point $\left(x_{1}, x_{2}, \ldots, x_{n}\right)$ of which satisfying $x_{1} \ldots \ldots x_{n}$, by four different

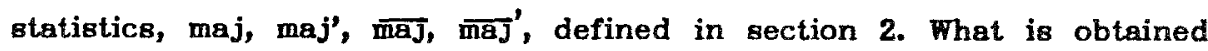
are determinantal expressions (Theorems 1 to 4 in section 6). The method of proof we give is combinatorial and is inspired by Zeilberger's [13] reflection proof for the case $q=1$ and a pseudo-reflection proof for the case $n=2$ by Fürlinger and Hofbauer [3, Lemma on p.255].

There is a bijection between lattice paths from 0 to $\lambda$ and permutations of the multiset $\left\{1^{\lambda_{1}}, 2^{\lambda_{2}}, \ldots, n^{\lambda_{n}}\right\}$ (this symbol denotes the 
multiset with objects $[1,2, \ldots, n]$ and multiplicities $\left.\left(\lambda_{2}, \lambda_{2}, \ldots, \lambda_{n}\right)\right)$. Namely, each $k$ in a multiset permutation is interpreted as step in the $x_{k}$-direction. MacMahon calls the permutations corresponding to those paths, whose points satiafy $x_{1} \cdots \cdots x_{n}$, "lattice permutations". He is lead to consider lattice permutations when evaluating the generating function for plane partitions of a given shape $\lambda[8$, Bec.IX, ch.III; sec.X]. What has to be done is to count lattice permutations by the major-statistics; i.e., to evaluate the weighted sum $\sum q^{\operatorname{maj}} \pi$, where the sum is over all lattice permutations $n$ of the multiset $\left[1^{\lambda_{1}}, 2^{\lambda_{2}}, \ldots, n^{\lambda_{n}}\right]$. Later, MacMahon's method was formalized and generalized by Stanley [10, Corollaries 5.3 and 7.2]. Thus, wanting to find a certain partition generating function, one has to count a specific collection of permutations by the major index.

In section 3 we shall see that in this sense evaluation of the generating functions for plane partitions, column-strict plane partitions, reverse plane partitions and column-strict reverse plane partitions of a given shape $\lambda$ is done by solving the problem of counting lattice permutations by maj, maj', $\overline{\mathrm{maj}}$ ' and $\overline{\mathbf{m a j}}$, in that order, respectively. Thus, as corollaries of our lattice path enumeration results, we obtain determinantal expressions even for the generating functions for skew plane partitions, etc., of a given shape $N \boldsymbol{\mu}$. The expressions for column-strict and column-strict reverse skew plane partitions (Corollary 9) are well-known and may be found in [10, p.82 for $u=\infty]$. The expressions for ordinary and reverse skew plane partitions (Corollaries 7 and 8) seem to be new. The special case $\mu=0$ of ordinary plane partitions was solved by MacMahon [8] by inductive arguments which are not very rigorous. In the remaining three cases for $\mu=0$ the determinantal expressions can be aimplified, which leads to the well-known hook-formulae for the generating functions of column-strict, reverse and column-strict reverse plane partitions of a given shape $\lambda$ [11, Theorem 15.3 for $m=\infty$, Propositions 18.3 and 18.4]. For these results there are proofs by Schur functions [11], a combinatorial proof explaining the determinantal expressions [5] and various bijective proofs explaining the occurence of the hook-lengths $[2,4,6,9]$.

Our method of proof offers a new combinatorial explanation for the determinantal expressions for all of the above stated four kinds of generating functions, even in the case of the various kinds of skew 
plane partitions. MacMahon's determinant is generalized and given combinatorial view.

In section 2 we give all relevant definitions. The connection between lattice path enumeration and generating functions for skew plane partitions is explained in section 3. Section 4 contains a recapitulation of Zeilberger's [13] reflection proof. In section 5 we give the bijections which replace the reflection in Zeilberger's proof. These bijections, in section 6, are used to obtain the promised determinantal expressions. Finally, in section 7 , these results are transferred to obtain the corresponding generating functions for the various kinds of skew plane partitions.

2. DEFINITIONS. We use the usual multidimensional notation. If $\lambda=\left(\lambda_{1}, \lambda_{2}, \ldots, \lambda_{n}\right), \quad \mu=\left(\mu_{1}, \mu_{2}, \ldots, \mu_{n}\right), \quad$ then $\quad|\lambda|=\lambda_{1}+\lambda_{2}+\cdots+\lambda_{n}, \quad \lambda+\mu=$ $=\left(\lambda_{2}+\mu_{2}, \lambda_{2}+\mu_{2}, \ldots, \lambda_{n}+\mu_{n}\right)$ and $\left(\begin{array}{c}|\lambda| \\ \mu\end{array}\right)=|\lambda| ! / \mu_{1} ! \mu_{2} ! \cdots \mu_{n} !$. If $\lambda_{1} m_{\mu_{1}}$ for $\stackrel{F}{=} 1,2, \ldots, n$ we write $\lambda \geq \mu$.

Let $q$ be an indeterminate. The $q$-notations we need are $[\alpha]=\left(1-q^{\alpha}\right)$, $[m]:=[m] \cdot[-1] \cdots[1],[0] !=1$ and $\left[\begin{array}{c}|\lambda| \\ \mu\end{array}\right]=[|\lambda|] ! /\left[\mu_{1}\right] !\left[\mu_{z}\right] ! \cdots\left[\mu_{n}\right] !$.

Let $S_{n}$ denote the symmetric group of order $n$. Given of $S_{n}$ we write $\lambda_{\sigma}$ for $\left(\lambda_{\sigma}(1), \lambda_{\sigma(2)}, \ldots, \lambda_{\sigma}(n)\right) . D_{n}$ stands for the set of all $n$-tupels of integers $\lambda$ with $\lambda_{1} \geqslant \lambda_{2} \geqslant \cdots>\lambda_{n}$. For the set of all permutations of the multiset with objects $\{1,2, \ldots, n\}$ and multiplicities $\mu=\left(\mu_{2}, \mu_{2}, \ldots, \mu_{n}\right)$ we write $S(\mu)$. Given a multiset permutation $\pi_{n} \pi_{1} \pi_{2} \ldots \pi_{p}, \quad \pi_{1} \varepsilon[1,2, \ldots, n]$, we introduce difference functione $d_{j}(\pi, i)$ for $j=1,2, \ldots, n-1$ :

$$
d_{j}(n, i)=\mid\left[r, r \in i \text { and } n_{r}=j\right\}|-|\left\{r, r i \text { and } n_{r}=j+1\right] \mid \text {. }
$$

$d_{j}(x, i)$ is the difference between the number of $j 8$ and the number of $(j+1)$ 's in $\pi_{1}, \ldots \pi_{i}$. Obviously $d_{j}(n, 0)=0$.

In this paper, a latice path $p=(p(0), p(1), \ldots, p(p))$ will be $a$ finite sequence of points in the integer lattice $Z^{n}$ with positive unit stepk, i.e. for $i=0, p(i+1)-p(i)=E_{j}$, for some $j, 1 \leqslant j \neq n$. $E_{j}$ stands for the vector with the $j$-th coordinate being equal to 1 , all other being equal to 0. $[p(i)]_{k}$ will denote the $k$-th coordinate of the $i$-th point on the path p. A lattice path $p$ from $\mu$ to $\lambda$ can be symbolized by a pair $(\mu, \pi)$, where $\mu=p(0)$ and $\pi \varepsilon S(\lambda-\mu)$. $x$ is obtained by proceeding along the path, starting at its first point $\mu$, and successively writing a for each step 
in the $x_{k}$-direction. To be precise, $\pi_{n}=\pi_{1} \pi_{2} \ldots \pi_{p}$, where $p=|\lambda-\mu|$ and $\pi_{i}=k$ if and only if $p(i)-p(i-1)=E_{k}$. Given a path $p$ in the form $(\mu, \pi)$, the points of $p$ are determined by

$$
p(i)=\mu+\sum_{k=1}^{n} E_{k} \cdot \mid\left[r \cdot 1 \leqslant r \leqslant p \text { and } \pi_{r}=k\right] \mid .
$$

To give an example, take $\mathrm{n}=3$ and $p_{0}=((1,2,1),(1,2,2),(1,2,3),(2,2,3)$, $(2,3,3),(3,3,3))$. Then $p_{0}$ is symbolized by the pair $((1,2,1), 33121)$.

The set of all lattice paths from $\mu$ to $\lambda$ is denoted by $M(\mu \rightarrow \lambda)$. We write $M(\mu \rightarrow \lambda)^{+}$for the set of all lattice paths from $\mu$ to $\lambda$ whose points all lie in $D_{n}$. For $\mu, \lambda \varepsilon D_{n}$ the set $M(\mu \rightarrow \lambda)^{+}$is equal to the set of all lattice paths from $\mu$ to $\lambda$ not touching any one of the hyperplanes

$$
x_{1}-x_{1+1}=-1, \quad \dot{=1}, 2, \ldots, n-1
$$

Let $H_{1}$ denote the hyperplane $x_{1}-x_{1+1}=-1$ and $R_{1}$ the reflection at $H_{1}$. $M(\mu \rightarrow \lambda)^{-}$stands for the set of all lattice paths from $\mu$ to $\lambda$ which touch at least one of the hyperplanes in $(2.2)$. Obviously $M(0 \rightarrow \lambda)^{+}$, or better $[n: p=(0, \pi) \varepsilon M(0 \rightarrow \lambda)+\}$, is the set of all lattice permutations.

Next, for a given multiset permutation $\pi_{n}=\pi_{1} \pi_{2} \ldots \pi_{p}$, we define the "down-set" $D(x)$ by

$$
D(\pi)=\left[r: \pi_{r}{ }^{2} \pi_{r}+1,16 r+p-1\right],
$$

and the four statistics maj, maj', $\overline{\mathrm{maj}}, \overline{\mathrm{maj}}$,

$$
\begin{aligned}
& \operatorname{maj} x={ }_{i=1}^{P-1} i \cdot x\left(\pi_{t}+\pi_{i+1}\right), \\
& \operatorname{maj}^{\prime} x={ }_{i=1}^{P} \sum_{i}^{-1} i \cdot x\left(\pi_{i}<\pi_{i}+1\right) \text {, } \\
& \overline{\operatorname{maj}} \times=\sum_{i=2}^{p}(p-i+1) \cdot x\left(n_{i}-1<n_{1}\right) \text {, } \\
& \overline{\overline{m e j}}, \pi=\sum_{i=2}^{p}(p-i+1) \cdot x\left(\pi_{i}-1>_{i}\right) .
\end{aligned}
$$

$(x(A)=1$ if $A$ is true, and $x(A)=0$ otherwise.) maj is the familiar major-statistics, counting the sum of descents, meaning

$$
\operatorname{maj} \pi=\sum_{1 \varepsilon D(\pi)} i \text {, }
$$

maj' counts the sum of ascents. $\overline{\mathrm{maj}}$ can be interpreted as maj "from the back", $\overline{\text { maj }}$ can be viewed as maj" "from the back".

Having in mind the representation of a lattice path $p$ by a pair $(\mu, x)$, we extend these statiotics to lattice paths by defining maj $p=\operatorname{maj} n$, 
etc., if $p=(\mu, \pi)$.

Finally we introduce the generating functions

$$
\begin{aligned}
& \Re(\mu \rightarrow \lambda ; q)=\sum_{p} q^{\text {maj } p,} \\
& F^{\prime}(\mu \rightarrow \lambda ; q)=\sum_{p} q^{m a j^{\prime} p}, \\
& \bar{F}(\mu \rightarrow \lambda ; q)=\sum_{p} q^{\overline{m a j} p,} \\
& \bar{F}^{\prime}(\mu \rightarrow \lambda ; q)=\sum_{p} q^{\overline{m a j}} p,
\end{aligned}
$$

where the sums are over all p\&M( $\rightarrow \lambda)$. Analogously,

$$
F(\mu \rightarrow \lambda ; g)^{+}=\sum_{\mathbf{p}} \phi^{m a j} p, \text { etc. }
$$

where the sum is over all $p \varepsilon M(\mu \rightarrow \lambda)^{+}$, and

$$
F(\mu \rightarrow \lambda ; q)^{-}=\sum_{p} q^{\operatorname{maj} p}, \text { etc. }
$$

where the sum is over all $p \& M(\mu \longrightarrow \lambda)^{-}$.

\section{GENERATING FUNCTIONS FOR PLANE PARTITIONS AND ENUMERATION} OF LATTICE PATHS. A plane partition of shape $\lambda$, where $\lambda \varepsilon D_{n}$ and $\lambda \geq 0$, is an array of positive integers

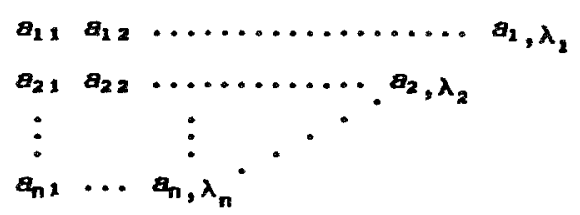

with entries decreasing in rows and columns. A skew plane partition of shape $\lambda / \mu$, where $\lambda, \mu \varepsilon D_{n}$ and $\lambda \lambda_{\mu}$, is an array of positive integers of the form

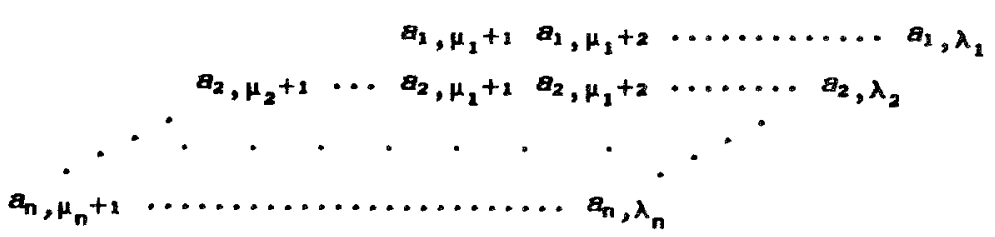

with entries decreasing in rows and columns. A column-strict (skew) plane partition is a (skew) plane partition with strictly decreasing entries in each column. A reverse (skew) plane partition is an array of 
positive integers of the form (3.1) (or (3.2), respectively) with entries increasing in rows and columns, a column-strict reverse (skew) plane partition is a reverse (skew) plane partition with strictly increasing entries in each column.

The weight $w(\Pi)$ of a (skew) plane partition is the sum of all its entries. The generating function for plane partitions of a given shape $\lambda$ is $\Sigma q^{w(\Pi)}$, where the sum is over all plane partitions $I$ of shape $\lambda$. Generating functions for column-strict (skew) plane partitions, etc., of a given shape $\lambda$ (or $\lambda / \mu$, respectively) are defined analogously.

In [10] Stanley considers partitions on a partial ordered set $P$ endowed with a labeling $\omega$, called $(P, \omega)$-partitions. His important theorem [10, Corollaries (5.3) and (7.2)] says that the generating function $U(P, \omega ; q)$ of $(P, \omega)$-partitions can be written in the form

$$
u(P, \omega ; q)=\frac{M P, \omega ; q)}{(1-q)\left(1-q^{2}\right) \cdots\left(1-q^{p}\right)}
$$

with

$$
M(P, \omega ; q)=\sum_{\sigma} q^{\mathrm{maj} \sigma}
$$

where the sum is over all permutations $\sigma$ of the $\omega$-separator [10, p.17] of $P$. Here, $p$ denotes the cardinality of $P$.

To obtain the case of plane partitions in this general theorem, for $P$ we have to take the set

$$
P(\lambda)=\left\{(i, j) \varepsilon \mathrm{N}^{2}: 1 \leqslant i \leqslant n \text { and } 1 \leqslant j \leqslant \lambda_{i}\right\}
$$

for a given $\lambda E D_{n}, \lambda>0$ and $|\lambda|=p . P(\lambda)$ becomes a poset by

$$
\left(i_{1}, j_{1}\right) \leftarrow\left(i_{2}, j_{2}\right) \text { if and only if } \quad i_{2} \leqslant i_{2} \text { and } j_{1} \leqslant j_{2}
$$

Take the labeling

$$
\omega(i, j)=\sum_{t=1}^{i-1} \lambda_{t}+j
$$

then there is a bijection between the set of $(P(\lambda), \omega)$-partitions and the set of plane partitions of shape $\lambda$, namely, by adding 1 to each of the parts of a $(P(\lambda), \omega)$-partition. Thus, by (3.3), the generating function for plane partitions of shape $\lambda$ is equal to

$$
\phi^{p} \frac{M(P(\lambda), \omega ; q)}{(1-q)\left(1-q^{2}\right) \cdots\left(1-q^{p}\right)}
$$


with

$$
M(\lambda), \omega ; q)=\sum_{\sigma} q^{\mathrm{maj} \sigma}
$$

where the surn is over all permutations of the w-separator of $P(\lambda)$. Given $\sigma=\sigma_{1} \sigma_{2} \ldots \sigma_{p}$, being an element of the $\omega$-separator of $P(\lambda)$, we define a multiset permutation $n=\pi(\sigma)=\pi_{1} \pi_{2} \ldots \pi_{p}$ by

$$
\pi_{1}=k \text { if and only if } \sum_{t=1}^{k-2} \lambda_{t}<\sigma_{1} \leqslant \sum_{t=2}^{k} \lambda_{t} \text {. }
$$

By definition $\pi \varepsilon S(\lambda)$. It is not difficult to see that since $\sigma$ is an element of the w-separator of $P(\lambda)$, maj $\pi=$ maj $\sigma$ and $\pi$ is a latice permutation, or in terms of paths $(0, \pi) \varepsilon M(0 \rightarrow \lambda)+$. For illustration we give an example. Let $m=3, \lambda=(3,2,1)$. Consider the following linear extension of the partial order (3.4) on $P(\lambda)$ :

$$
(1,1)<(2,1)<(1,2)<(1,3)<(3,1)<(2,2) \text {. }
$$

The corresponding $\sigma$ is $\omega(1,1) \omega(2,1) \omega(1,2) \omega(1,3) \omega(3,1) \omega(2,2)=142365$. By (3.6) we get $x=121132$. Obviously we have $\operatorname{maj} \pi=\operatorname{maj} \theta=7$, and $n$ is a lattice permutation.

Summarizing, these considerations furnish an alternative expression for $W(P(\lambda), \omega ; q)$ :

$$
\mathscr{H}(\lambda), \omega ; q)=\sum_{p} q^{\operatorname{maj}} p
$$

where the sum is over all paths $p \varepsilon M(0 \rightarrow \lambda)^{+}$.

More generally, to obtain skew plane partitions of given shape $N / \mu$, $\lambda, \mu \varepsilon D_{n}, \lambda \geq \mu$ and $|\lambda-\mu|=p$, we define

$$
P(\lambda / \mu)=\left[(i, J) \in N^{2}: 16 j \leqslant n \text { and } \mu_{1}<j \lambda_{i}\right]
$$

with order relation defined in (3.4), and with labeling

$$
\omega_{0}(i, j)=\sum_{t=1}^{1-1}\left(\lambda_{t}-\mu_{t}\right)+\left(j-\mu_{i}\right) .
$$

The $\left(P(\lambda / \mu), \omega_{0}\right)$-partitions are skew plane partitions. (The case of ordinary plane partitions comes out for $\mu=0$ ) Then, analogously, the generating function for skew plane partitions of shape $\lambda / \mu$ can be written

$$
q^{p} \frac{m\left(P(\lambda / \mu), \omega_{0} ; q\right)}{(1-q)\left(1-q^{2}\right) \cdots\left(1-q^{p}\right)}
$$


with

$$
\left.M(\lambda / \mu), \omega_{0} ; q\right)=\sum_{p} q^{\operatorname{maj} p}
$$

where the gum is over all $p \in M(\mu \rightarrow \lambda)^{+}$.

For the labeling

$$
\omega_{c}(i, j)=\sum_{t=i+1}^{n}\left(\lambda_{t}-\mu_{t}\right)+\left(j-\mu_{t}\right)
$$

the $\left(P(\lambda / \mu), \omega_{c}\right)$-partitions are column-strict skew plane partitions. Similarly, one obtains the generating function for column-gtrict plane partitions of shape $\lambda / \mu$ to be

$$
q^{p} \frac{M P(\lambda / \mu), \omega c ; q)}{(1-q)\left(1-q^{2}\right) \cdots\left(1-q^{p}\right)}
$$

with

$$
H(P(\lambda / \mu), \omega c ; g)=\sum_{p} q^{m a j ' p}
$$

where the gum is over all $p \in M(\mu \rightarrow \lambda)^{+}$.

Let $\overline{P(\lambda / \mu)}$ denote the the poset with same basic set as $P(\lambda / \mu)$, i.e., the right-hand side of (3.7), but with reversed partial order, meaning

$$
\left(i_{1}, j_{1}\right) \leqslant\left(i_{2}, j_{2}\right) \text { if and only if } i_{1} \geqslant i_{2} \text { and } j_{1} \geqslant j_{2}
$$

For the labeling

$$
\omega_{r}(i, j)=\sum_{t=1+1}^{n}\left(\lambda_{t}-\mu_{t}\right)+\left(\lambda_{1}-j+1\right)
$$

we get that the generating function for reverse skew plane partitions of shape $\lambda / \mu$ is equal to

$$
q \frac{\left.M \overline{P(\lambda / \mu)}, \omega_{r} ; q\right)}{(1-q)\left(1-q^{2}\right) \cdots\left(1-q^{p}\right)}
$$

with

$$
M \overline{P(\lambda / \mu)}, \omega_{r i q}=\sum \overline{q^{\mathrm{ag}}}, p
$$

For the labeling

$$
\omega_{C r}(i, j)=\sum_{t=1}^{1-1}\left(\lambda_{t}-\mu_{t}\right)+\left(\lambda_{1}-j+1\right)
$$

we obtain that the generating function for column-strict reverse skew 
plane partitions of shape $\lambda / \mu$ is

$$
q^{p} \frac{\left.m \overline{P(\lambda / \mu)}, \omega_{c r i} q\right)}{(1-q)\left(1-q^{2}\right) \cdots\left(1-q^{p}\right)}
$$

where

$$
\left.M \overline{P(\lambda / \mu)}, \omega_{c r} q\right)=\sum_{p} \overline{\bar{p}^{\bar{Q} \mathrm{~J}} p} .
$$

In both cases the sum is over all $p \in M(\mu \rightarrow \lambda)^{+}$.

4. ZEILBERGER'S REPLECTION PROOF. Let $\lambda, \mu \in D_{n}$ and $\lambda \geqslant \mu$. In this section, in order to make the following more transperent, we recapitulate Zeilberger's [13] method of counting the number of elements of $M(O \rightarrow \lambda)+$. A little bit more generally, we extend his method to evaluate the number of elements of $M(\mu \rightarrow \lambda)^{+}$, which, recalling the definition of $F(\mu \rightarrow \lambda ; q)^{+}$, is seen to be equal to $F(\mu \rightarrow \lambda ; 1)^{+}$.

For the number of all lattice paths from $\mu$ to $\lambda, F(\mu \rightarrow \lambda ; 1)$, holds

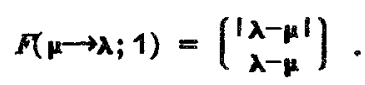

Following Zeilberger we define

$$
e_{\sigma}=(1-\sigma(1), \ldots, i-\sigma(i), \ldots, b-\sigma(n))
$$

The crucial point in the proof is to construct a bijection between

$$
\operatorname{Ueven}_{\sigma} M\left(\mu_{\sigma}+e_{\sigma} \rightarrow \lambda\right)^{-} \text {and } \sigma \operatorname{odd}^{U} M\left(\mu_{\sigma}+e_{\sigma} \rightarrow \lambda\right)^{-}
$$

which proves

$$
\sigma \sum_{\text {even }} F\left(\mu_{\sigma}+e_{\sigma} \rightarrow \lambda ; 1\right)^{-}=\sum_{\sigma d d} \Gamma\left(\mu_{\sigma}+e_{\sigma} \rightarrow \lambda ; 1\right)^{-}
$$

This is done by application of the reflection principle. Consider a lattice path of $M\left(\mu_{\sigma}+e_{\sigma} \rightarrow \lambda\right)$. Let $H_{j}$ be the last hyperplane of $(2.2)$ the path meets. Since reflection with respect to $H_{j}$ has the effect

$$
R_{j}\left(x_{1}, \ldots, x_{j}, x_{j+1}, \ldots, x_{n}\right)=\left(x_{1}, \ldots, x_{j+1}-1, x_{j}+1, \ldots, x_{n}\right) \text {, }
$$

we have

$$
R_{j}\left(\mu_{\sigma}+e_{\sigma}\right)=\mu_{\sigma}(j, j+1)+e_{\sigma}(j, j+1) .
$$


$((j, j+1)$ is the transposition which exchanges $j$ and $j+1$.$) Therefore,$ replacing the portion of the path until the last meeting with $H_{j}$ by its reflection with respect to $H_{j}$, turns this path into a path from

$\mu_{\sigma}(j, j+1)+e_{\sigma}(j, j+1)=$

$=\left(\mu_{\sigma}(1)+1-\sigma(1), \ldots, \mu_{\sigma}(j+1)+j \sigma(j+1), \mu_{\sigma}(j)+j+1-\sigma(j), \ldots, \mu_{\sigma}(n)+n-\sigma(n)\right)$

to $\lambda$, touching $H_{j}$. This defines the desired bijection, hence (4.2) holds. Since, because of $\mu \varepsilon D_{n}$, for $\sigma^{\neq}$id we have $\left(\mu_{\sigma}+e_{\sigma}\right) \in D_{n}$, we get

$$
\left.M \mu_{\sigma}+\varepsilon_{\sigma} \rightarrow \lambda\right)^{-}=M\left(\mu_{\sigma}+e_{\sigma} \rightarrow \lambda\right), \quad \text { if } \sigma \neq i d .
$$

Therefore, by consecutive use of (4.2), (4.5), and (4.1),

$$
\begin{aligned}
& F(0 \rightarrow \lambda ; 1)^{+}=F(0 \rightarrow \lambda ; 1)-F(0 \rightarrow \lambda ; 1)^{-} \\
& =F(0 \rightarrow \lambda ; 1)+\sum_{I_{1 d}}(-1)^{8 g n} \sigma F\left(\mu_{\sigma}+\varepsilon_{\sigma} \rightarrow \lambda ; 1\right)^{-} \\
& =\sum_{\sigma \varepsilon S_{n}}(-1)^{\operatorname{sgn} \sigma} \Gamma_{\left(\mu_{\sigma}+e_{\sigma} \rightarrow \lambda ; 1\right)} \\
& =\sum_{\sigma \varepsilon S_{n}}(-1)^{\operatorname{sgn} \sigma} \cdot\left(\begin{array}{c}
|\lambda-\mu| \\
\lambda-\mu_{\sigma}-e_{\sigma}
\end{array}\right) \\
& =|\lambda-\mu| ! \operatorname{det}\left(1 /\left(\lambda_{\mathrm{s}}-s-\mu_{t}+t\right) !\right) \text {. }
\end{aligned}
$$

REMARK. Zeilberger was wrong when taking the first hyperplane a lattice path touches and reflecting the part of the path until that meeting, because this defines no bijection. We give a aimple counter-example. Let $n=3, \mu 0$ and $\lambda=(1,1,1)$. For $\sigma(2,3)$ we have $e_{0}=(0,-1,1)$. The lattice path $((0,-1,1), 221)$ first meets the hyperplane $x_{2}-x_{3}=1$ (namely after its first step), the reflection with respect to $x_{2}-x_{3}=-1$ of the portion of the path until the meeting point $(0,0,1)$ gives the path $(0,321)$. Similarly, this procedure turns $(e(1,2)=((-1,1,0), 311)$ into (0, 321), the same path. Hence, this cannot be a bijection. The reason is that the property "meeting $H_{j}$ as first of all hyperplanes of (2.2)" is not preserved under reflection until the first meeting point. (In our example $((-1,1,0), 311)$ first meets $H_{2}$, but its image under reflection, $(0,321)$, first meets $H_{2}$ ). This mistake is corrected (as done above) by taking the last meeting, for "meeting $H_{j}$ as last of all hyperplanes of (2.2)" is preserved under reflection until the last meeting point. Therefore this mapping is an involution and, hence, a bijection.

The same mistake was made by Watanabe and Mohanty [12], who refine Zeilberger's reflection proof. But everything remains true after 
replacing lines 21-23 on page 282 of [12] by "for the lasi time, only any one of the hyperplanes $[\lg (k)]: k \varepsilon S]$ out of $[\lg (k)]: k \in[1,2, \ldots, n-1)]$. (Such a path may met $[g(k)]$, $k \ell S$, but only before meeting at least one $[g(k)]$, k\&S)."

In our context the above reflection procedure must be modified because, when reflecting a part of a lattice path, nothing can be said about the major of its image. Therefore, in order to generalize the reflection proof to $q \neq 1$, in the next section we give maps which send the initial point of a lattice path to the same point as the reflection procedure would do, but, what is more, are (almost) maj-(maj'-, $\overline{\text { maj-, }}$ maj'-)preserving. The remaining steps in the above proof can be transferred almost verbatim. This will be done in section 6.

5. THE BIJECTIONS. As mentioned above, in Zeilberger's proof given in the preceding section, we do not really need the reflection. What we need is a bijection which sends a lattice path $p$ from $\mu$ to $\lambda$ to a lattice path $\bar{p}$ from $R_{j}(\mu)$ to $\lambda$, where $H_{j}$ is the last hyperplane of (2.2) the lattice path meets. Regarding (4.3), if $\mu_{j} \|_{j}+1$, we see that, changing $\left(\mu_{j}-\mu_{j+1}+1\right)$ of the steps in the $x_{j+1}$-direction in the path $p$ into steps in the $x_{j}$-direction, we obtain a lattice path from $R_{j}(\beta)$ to $\lambda$. Analogously, if $\mu_{j}<\mu_{j}+1$, changing $\left(\mu_{j}+2-\mu_{j}-1\right)$ of the steps in the $x_{j}$-direction into steps in the $x_{j+2}$-direction, turns $p$ into $a$ path from $R_{j}(\mu)$ to $\lambda$.

Next, for a fixed $j$, we introduce two auxiliary maps, $L_{j}$ ("lower") and $R_{j}$ ("raise"). $L_{j}$ has the effect of changing aingle step in the $x_{j+2}$-direction into a step in $x_{j}$-direction, $R_{j}$ has the reverse effect. $\left(\mu_{j}-\mu_{j+1}+1\right)$-time application of $L_{j}$ or $\left(\mu_{j+1}-\mu_{j}-1\right)$-time application of $R_{j}$, respectively, depending on whether $\mu_{j} \neq_{j}+1$ or not, leade to the desired bijection.

DEFINITION 1. Let $j$ be a fixed integer, $16 j \in n-1$, and let $n=n_{1} \ldots \pi_{p}$ be a multiset permutation where the function $i d_{j}(x, i)$ does not reach ite minimal value at $j=0$ or $j=p$. Let $k$ be the smallest integer $1 \leqslant k \leqslant p-1$, where the function $i \rightarrow d_{j}(x, i)$ reaches its minimal value, in other words

$$
\begin{array}{lll}
i<k & \text { implies } & d_{j}(\pi, i)>d_{j}(\pi, h) . \\
i \geq k & \text { implies } & d_{j}(\pi, i) \geq d_{j}(\pi, k) .
\end{array}
$$

Then $\pi_{k}=j+1$. This given, we define the "lower" function $L_{j} b y$ : 
(A) If * satisfies

$$
n_{e}<\pi_{e+1}>\ldots+n_{f} \leq \pi_{f+1}>\ldots+\pi_{k}-1>j+1>n_{k+1}>\ldots>\pi_{g} \leq \pi_{g}+1 \text {, }
$$

where $0 \leqslant e+1<f 1<g^{6} p$, and

$$
\begin{array}{ll}
\text { either (a) } f+1<k^{<} g \\
\text { or } & \text { (b) } k=f+1 \geq 2 \text { and } n_{f}<n_{f+2} \\
\text { or } & \text { (c) } k=g \text { and } n_{g-1} \leqslant n_{g+1},
\end{array}
$$

then

$\boldsymbol{L}_{\mathrm{j}}(\pi)=\pi_{1} \ldots \pi_{\mathrm{q}} \pi_{\mathrm{g}+1} \ldots \pi_{r-1} j \pi_{r} \ldots \pi_{f} \pi_{f+1} \ldots \pi_{k-1} \pi_{k+1} \ldots \pi_{g} \pi_{g+1} \ldots \pi_{p}$,

where $r$ is minimal with

$$
A_{\pi_{r}} \text { and et } 1 \leqslant r \in f \text {, }
$$

if there is none, $p=A 1$.

(B) If satisfies

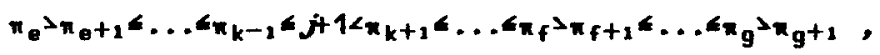

where $1 \leqslant e+1<f<g^{\leq} p$, and

$$
\begin{array}{ll}
\text { either (a) } e+1<k<f \\
\text { or } & \text { (b) } k=e+1 \text { and } n_{0} x_{0+2} \\
\text { or } & \text { (c) } k=f \text { and } x_{f-1}+x_{f+1},
\end{array}
$$

then

$$
\begin{aligned}
& L_{j}(x)=n_{1} \ldots n_{e} n_{e+1} \ldots n_{k-1} n_{k+1} \ldots n_{f} n_{f+1} \ldots \pi_{r-1} j n_{r} \ldots \pi_{g} n_{g}+1 \ldots \pi_{p} \text {, } \\
& \text { where } r \text { is minimal with }
\end{aligned}
$$

$$
j \bar{k}_{r} \text { and } f+1 \leqslant r k g \text {, }
$$

if there is none, $r=g+1$.

(By convention, if $i j$, the symbol $\pi_{1} \ldots \pi_{j}$ means the empty word.)

REMARKS. (1) Given $j$ and a multiset permutation * satisfying the condition required in Definition 1 , there are exactly five cases:

$$
\begin{aligned}
& n_{k-1}>j+1>n_{k+1}, 2 \leqslant k^{<p} \\
& n_{k-1} \leqslant j+1<n_{k+1}, 1 \leqslant k<p \\
& n_{k-1}>j+1<n_{k+1}, 2 \leqslant k<p \\
& n_{k-1} \leqslant j+1 n_{k+1}, 2 \leqslant k<p \\
& j+1>n_{2} \text { and } k=1 .
\end{aligned}
$$

$\left(j+1=x_{k+2}\right.$ is impossible, because then $d_{j}(x, k+1)=d_{j}(x, k)-1$, in contradiction to the minimality of $d_{j}(n, k)$.)

Case (5.3) belongs to case $(A)(a)$ in Definition 1, (5.4) to (B)(a). If 
$\pi_{k-1} \leqslant_{k+1}$, (5.5) belongs to case $(A)(c)$, otherwise it belongs to (B) (b). Similarly, if $\pi_{k-1}<\pi_{k+1},(5.6)$ belongs to $(A)(b)$, otherwise it belongs to (B)(c). Finally, (5.7) belongs to (B)(c). Therefore Definition 1 covers all possible cases; moreover, given multiset permutation, this multiset permutation uniquely belongs to only one of the cases (A) or (B). Thus $L_{\mathrm{y}}$ is well-defined.

(2) To be precise, the inequality chains (5.1) and (5.2) should be understood as short-hand writings in the following cases:

$k=f+1$ in (5.1) means the inequality chain $x_{e} 4 x_{t}+1+\ldots+x_{f} \leqslant j+1 x_{k+1}>\ldots+x_{g} x_{g+1} . \quad k=g$ in $(5.1)$, and $k=\theta+1$ or $k=f$ in (5.2) should be understood analogously.

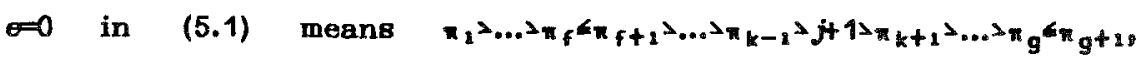
analogous meanings have 0 in $(5.2)$ and $g=p$ in $(5.1)$ or (5.2).

$f=0$ in (5.1) means $x_{1}>\ldots x_{k-1}>j+1>\pi_{k+1}>\ldots>x_{g} x_{g}+2$. In this case $L_{j}(x)$ should be understood as

$$
L_{j}(n)=j \pi_{2} \ldots \pi_{k-1} \pi_{k+1} \ldots \pi \pi g+2 \ldots \pi_{p} .
$$

$f=p$ in (5.2), meaning $\pi_{a}>\pi_{e}+x \leqslant \ldots \leqslant \pi_{k-1} \leqslant j+1<\pi_{k+1} \leqslant \ldots \kappa_{p}$, is impossible, because then $d_{j}(x, k)=d_{j}(x, p)$, which contradicts non-minimaity of $d_{j}(x, p)$.

(3) The following properties of $d_{j}$ hold:

$$
\begin{array}{ll}
d_{j}\left(L_{j}(x), i\right)=d_{j}(x, i), & \text { for } 0 \leqslant i<\min (r, h) \\
d_{j}\left(L_{j}(\pi), i\right)=d_{j}(\pi, i)+1, & \text { for } \min (r, k) \leqslant i<\max (r, k) \\
d_{j}\left(L_{j}(\pi), i\right)=d_{j}(\pi, i)+2, & \text { for } \max (r, k) \leqslant i \leqslant p .
\end{array}
$$

Since $x_{1} f\{j, j+1\}$ for $\min (r, k+1)<i<\max (r, k)$, we have

$$
d_{j}\left(L_{j}(\pi), r-1\right)=d_{j}(\pi, k)+1
$$

Together with $(5.8)-(5.10)$ and the definition of $k$ this leads to

$$
\begin{array}{lll}
i \leqslant r-1 & \text { implies } & d_{j}\left(L_{j}(\pi), i\right) \geq d_{j}\left(L_{j}(x), r-1\right), \\
i \geq r-1 & \text { implies } & d_{j}\left(L_{j}(\pi), i\right)>d_{j}\left(L_{j}(\pi), r-1\right) .
\end{array}
$$

In other words, $(r-1)$ is the largest integer where the function $i \rightarrow d_{j}\left(L_{j}(\pi), i\right)$ reaches its minimal value. Moreover, $d_{j}\left(L_{j}(\pi), p\right)$ is at least 2 larger than the minimal value of $i \rightarrow d_{j}\left(L_{j}(\pi), i\right)$. This bases on $(5.10)$ and (5.11), and the property $d_{j}(x, p) \geq d_{j}(x, k)+1$, required in Definition 1.

As corollary of (5.11) we get 


$$
\min _{i} d_{j}\left(L_{j}(\pi), i\right)=\min _{i} d_{j}(\pi, i)+1 .
$$

(4) $L_{j}$ has the important property

$$
\operatorname{maj}\left(L_{j}(\pi)\right)=\operatorname{maj} \pi-1
$$

(This is the reason why we called $L_{j}$ "lower" function.) For case (B) in Definition 1 this is clear from the definition of $L_{j}(\pi)$, since $D(x)=\{\ldots, e, f, g, \ldots\}$ and $D\left(L_{j}(x)\right)=\{\ldots e, f-1, g, \ldots\}$. This means, to obtain $D\left(L_{j}(x)\right)$, in $D(x)$ the element $f$ has to be replaced by $f-1$, which, by (2.3), proves (5.13). In case (A) we claim that $\pi_{r-1} \neq j$. Indeed, supposing $\pi_{r-1}=j$, because of $x_{i} k\{j, j+1\}$ for $r \leqslant j \leqslant k-1$, we would have $d_{j}(\pi, r-2)=d_{j}(\pi, k)$, in contradiction to the condition that $k$ is the smallest integer where $\mapsto d_{j}(\pi, i)$ reaches its minimal value. As $r$ is minimal with $j \pi_{r}$ and $\pi_{r-1}>\pi_{r}$, we conclude $\pi_{r-1}>j$. Consequently, $D(\pi)=[\ldots, e+1, \ldots, f-1, f+1, f+2, \ldots, g, \ldots.\} \quad \mathrm{D}\left(L_{j}(\pi)\right)=$ $=[\ldots, \theta+1, \ldots, f-1, f, f+2, \ldots, g, \ldots\}$. This means, to obtain $D\left(L_{j}(x)\right)$, in $D(x)$ the element $f+1$ has to be replaced by $f$, which again implies (5.13).

The next definition introduces the "raise" function $R_{j}$, which will turn out to be the inverse of $L_{\mathrm{j}}$.

DEFINITION 2. Let $j$ be a fixed integer, $1<j \leqslant n-1$, and let $\pi=\pi_{1} \ldots \pi_{p}$ be a multiset permutation where $d_{j}(\pi, p)$ exceeds the minimal value of the function $i d_{j}(x, i)$ by at least 2 . Let $(r-1)$ be the largest integer, $0<r-1 \leqslant p-1$, where $\rightarrow d_{j}(x, i)$ reaches its minimal value, meaning

$$
\begin{array}{lll}
i \leq r-1 & \text { implies } & d_{j}(x, i) \geq d_{j}(x, r-1) \\
i>r-1 & \text { implies } & d_{j}(x, i)>d_{j}(x, r-1) .
\end{array}
$$

Then $x_{r}=j$. This given, we define the "raise" function $R_{j}$ by:

(A) If satisfies

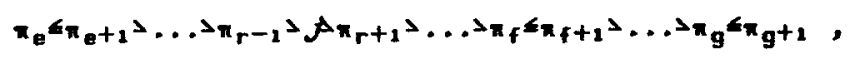

where $0 \leqslant e+1<f<g \leqslant p$, and

$$
\begin{array}{ll}
\text { either (a) e+1<p<f } \\
\text { or } & \text { (b) } r=e+1 \text { and } \pi_{0} \leqslant \pi_{a+2} \\
\text { or } & \text { (c) } r=f \text { and } \pi_{f-1} \leqslant \pi_{f+1},
\end{array}
$$

then

$R_{j}(\pi)=\pi_{1} \ldots \pi_{e} \pi_{e+2} \ldots \pi_{r-1} \pi_{r+1} \ldots \pi_{f} \pi_{f+1} \ldots \pi_{k-1} j+1 n_{k} \ldots \pi_{g} \pi_{g+1} \ldots \pi_{p}$, where $k$ is minimal with

$j+1 \times_{k}$ and $f+1 \leq k \leq g$, 
if there is none,

(B) If satisfies

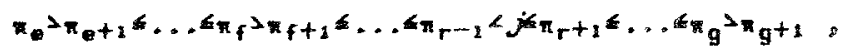

where $16 e+1<A 1<B^{*} p$, and

$$
\begin{array}{ll}
\text { either } & \text { (a) } A 1<r<g \\
\text { or } & \text { (b) } r=f+1 \text { and } n_{f} x_{f+2} \\
\text { or } & \text { (c) } r=g \text { and } n_{g-1} x_{g}+1
\end{array}
$$

then

$R_{j}(\pi)=n_{1} \ldots \pi_{k} \pi_{k+1} \ldots n_{k-2} j+1 n_{k} \ldots \pi_{q} \pi_{k+1} \ldots \pi_{r-1} n_{r}+8 \ldots \pi n_{g}+1 \ldots \pi_{p}$.

where $k$ is winimal with

$j+1<x_{k}$ and e+1kk*f,

if there is none, $k=f+1$.

REMARKS. (1) As above, it can be seen that Definition 2 covers all possible cases. In particular, the case $r=1$ belongs to case $(A)$ in Definition 2. If $j_{\pi_{3}}$, then it is the case $=0$ in $(A)(b)$, if $j *_{z}$, then it is the case $f=1$ in $(A)(c)$. In the latter case $R_{j}(x)$ should be understood as

$$
R_{j}(\pi)=\pi_{2} \ldots \pi_{k-1} j+1 \pi_{k} \ldots \pi \pi_{g}+2 \ldots \pi_{p} \text {. }
$$

$j=\pi_{r-1}$ is impossible, because then $d_{j}(x, r-2)=d_{j}(x, r-1)-1$, in contradiction to $d_{j}(x, r-1)$ being minimal.

(2) The inequality chains (5.14) and (5.15) should be understood as short-hand writings in a similar manner like (5.1) and (5.2). The case

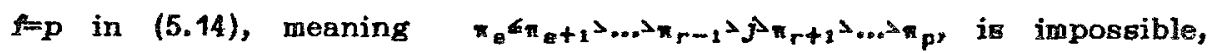
because then $d_{j}(\pi, p)=d_{j}(\pi, r-1)+1$, in contradiction to the condition that $d_{j}(x, p)$ exceeds the minimal value of $i d_{j}(n, i)$ by at least 2 .

(3) In a similar manner as above, it can be seen that $k$ is the smallest integer where the function $\rightarrow d_{j}\left(R_{j}(\pi), i\right)$ reaches its minimal value. Besides, $\rightarrow d_{j}\left(R_{j}(x), i\right)$ does not reach its minimal value at $=0$ or $\dot{f}=p$.

(4) $R_{j}$ has the property

$$
\operatorname{maj}\left(R_{j}(\pi)\right)=\operatorname{maj}_{n}+1
$$

which is proved quite similarly as $(5.13)$ above. $((5.16)$ is the reason for calling $R_{j}$ "raise" function".)

Regarding the remarks (3) after Definitions 1 and 2, respectively, we see 


$$
R_{j} \circ L_{j}=L_{j} \circ R_{j}=i d,
$$

which means that $R_{j}$ and $L_{j}$ are inverses of each other. In this sense we may write $R_{\mathrm{j}}=L_{\mathrm{j}}{ }^{-1}$.

Now we are in the position to construct the desired bijection 4 between

$$
\underset{\sigma}{\operatorname{Uven}} M\left(\mu_{\sigma}+e_{\sigma} \rightarrow \lambda\right)^{-} \text {and } \underset{\sigma d d}{\mathrm{U}} M\left(\mu_{\sigma}+e_{\sigma} \rightarrow \lambda\right)^{-}
$$

DEFINITION 3. Let $\lambda, \mu \varepsilon D_{n}, \lambda \geq \mu$, and let $p=(\mu, \pi)$ be a lattice path of $M(\mu \rightarrow \lambda)$-. Let $H_{J}$ be the last hyperplane of (2.2) p meets. Then + is defined by

$$
\Psi(p)=\left(R_{j}(\mu), L_{j}{ }^{\mu_{j}-\mu_{j+2}+1}(x)\right)
$$

REMARKS. (1) If the exponent $\left(\mu_{j}-\mu_{j+1}+1\right)$ in (5.18) is negative, by $L_{j} \mu_{j}-\mu_{j+1}+1$ we mean $R_{j}^{-\left(\mu_{j}-\mu_{j+1}+1\right)}$.

(2) To prove that is well-defined, we must show that $L_{j}$ is applicable on $\pi$ for $\left(\mu_{j}-\mu_{j+1}+1\right)$ times.

First we consider the case $\mu_{j} \neq_{j+1}$. Let $k$ be the smallest integer where $\leftrightarrow d_{j}(n, i)$ reaches its minimal value. Consider a path point $p(m)$, 1; $m<p$, where $p$ meets $H_{j}$, the hyperplane $x_{j}-x_{j+1}=-1$. Since $p$ starts in $\mu$, remembering (2.1), we clearly have $d_{j}(\pi, m)=\mu_{j+1}-\mu_{j}-1$, and therefore

$$
\min _{1} d_{j}(\pi, i) \div \mu_{j+1}-\mu_{j}-1
$$

If $L_{j}$ is applicable on for $t$ times we get by (5.12)

$$
\min _{1} d_{j}\left(L_{j} t(x), i\right)<\mu_{j}+i-\mu_{j}+t-1
$$

Since $\lambda \varepsilon D_{n}$, which in particular says $\lambda_{j} \lambda_{j}+1$, we get

$$
\begin{aligned}
d_{j}(x, p) & =\left(\lambda_{j}-\mu_{j}\right)-\left(\lambda_{j+1}-\mu_{j}+1\right) \\
& =\mu_{j+1}-\mu_{j}+\left(\lambda_{j}-\lambda_{j+1}\right) \\
& \geqslant \mu_{j}+1-\mu_{j},
\end{aligned}
$$

and after $t$-times epplication of $L_{j}$, if this is possible, using (5.10)

$$
d_{j}\left(L_{j} t(x), p\right)>\mu_{j}+1-\mu_{j}+2 t
$$

Comparison with (5.20) gives

$$
d_{j}\left(L_{j} t(x), p\right)>\min _{i} d_{j}\left(L_{j}^{t}(x), i\right)
$$


for all $t \geqslant 0$ where $L_{j} t(\pi)$ is defined. Besides, $d_{j}\left(L_{j} t(x), 0\right)=0$ for all $t \geqslant 0$. Together with (5.20) this leads to

$$
d_{j}\left(L_{j} t(x), 0\right)>\min d_{j}\left(L_{j} t(x), j\right)
$$

valid for all $t$ with $0 \leqslant t \leqslant \mu_{j}-\mu_{j}+1$. If $L_{j} t(\pi)$ is well-defined, (5.21) and (5.22), together, simply say that the minimum value of $\mapsto d_{j}\left(L_{j} t(x), j\right)$ is not reached at $\dot{t}=0$ or $\dot{F}=p$. Hence, $L_{j}$ is applicable on $L_{j}{ }^{t}(x)$ (if $L_{j}^{t}(x)$ is well-defined and $\left.0 \leqslant t<\mu_{j}-\mu_{j}+1\right)$. Because of $L_{j} t(\pi)$ being well-defined for $t=0$, inductively we deduce that $L_{j}$ is applicable on $a$ for $\left(\mu_{j}-\mu_{j+1}+1\right)$ times. This shows that is well-defined for $\mu_{j} \mu_{j}+1$. Similar arguments settle that + is well-defined also in the case $\mu_{j}<\mu_{j+1}$.

(3) We claim that the final point of $+(p)$ is $\lambda$. Since $p$ is a path from $\mu$ to $\lambda_{1}=$ is a multiset permutation being an element of $S(\lambda-\mu)$. Each application of $L_{j}$ turns a single $j+1$ into a $j$, and each application of $R_{j}=L_{j}^{-1}$ turns a single $j$ into a $j+1$. Therefore the number of $j s$ in $L_{j}{ }^{\mu_{j}-\mu_{j+1}+1}(x)$ is equal to $\left(\lambda_{j}-\mu_{j}\right)+\left(\mu_{j}-\mu_{j+1}+1\right)=\lambda_{j}-\mu_{j+1}+1$, the number of $(j+1)^{\text {s }} \mathrm{s}$ in ${L_{j}}^{\mu_{j}-\mu_{j+1}+1}(\pi)$ is equal to $\left(\lambda_{j+q}-\mu_{j+1}\right)-\left(\mu_{j}-\mu_{j+1}+1\right)=$ $=\lambda_{j+2}-\mu_{j}-1$. By (4.3) the $j$-th coordinate of $R_{j}(\mu)$ is $\mu_{j+2}-1$, the $(j+1)-$ th coordinate of $R_{j}(\mu)$ is $\mu_{j}+1$. Let $v$ be the final point of $(p)$. Then $v_{i}$ is equal to the $i$-th coordinate of $R_{j}(\mu)$ added to the number of $P^{\prime}$ in $L_{j}{ }^{\mu_{j}-\mu_{j+1}+1}(x)$. Together with the considerations above, this establishes $\sim \lambda$.

(4) Let $H_{j}$ be the last hyperplane of (2.2) the path $p=(\mu, \pi)$ meets. Let $p(m), 1 \leqslant m<p$, be the last point where $p$ meets $H_{j}$. Then $x_{m+1}=j$. Besides, $d_{j}(x, m)=\mu_{j+1}-\mu_{j}-1$ and $d_{j}(x, i)>\mu_{j+1}-\mu_{j}-1$ for all $i m$. Therefore for an integer $k$, where $d_{j}(x, k)$ is a minimum for the function $i d_{j}(\pi, i)$, must hold $k \leqslant m$. If $\mu_{j}<\mu_{j+1}-1$ we have

$$
d_{j}(\pi, 0)=0<\mu_{j+1}-\mu_{j}-1=d_{j}(\pi, \pi),
$$

therefore in this case even $k<m$. In view of this and looking at Definitions 1 and 2 , it is not difficult to see that for $i m$

$$
\left[L_{j}(\pi)\right]_{1}=\pi_{i} \text { and }\left[R_{j}(\pi)\right]_{i}=\pi_{i}
$$

From this fact we deduce, remembering Definition 3 and Remark (3) after it, that application of leaves the path-part between $p(m)$ and 
$p(p)$ unchanged. In particular, $p(m)$ also is a point of $\phi(p)$. Therefore the property "meeting $H_{j}$ as last of all hyperplanes of $(2.2)$ " is preserved under application of * Consequently,

$$
\begin{aligned}
& (+0)(p)=(+0+)((\mu, x)) \\
& =+\left(R_{j}(\mu), L_{j}{ }^{\mu_{j}}-\mu_{j+i}+1(x)\right) \\
& =\left(\left(R_{j} \circ R_{j}\right)(\mu),\left(L_{j}\left[R_{j}(\mu)\right]_{j}-\left[R_{j}(\mu)\right]_{j+1}+1 \circ L_{j} \mu_{j}-\mu_{j+1}+1\right)(\pi)\right) \text {. }
\end{aligned}
$$

But $\quad R_{j} \circ R_{j}=i d$, and, by $(4.3), \quad\left[R_{j}(\mu)\right]_{j}=\mu_{j}+1-1 \quad$ and $\left[R_{j}(\mu)\right]_{j+1}=\mu_{j}+1$. This turns (5.23) into

$$
(+\circ)(p)=p .
$$

In other words, is an involution.

(5) To see that is a bijection between

$$
\text { - Uven } M\left(\mu_{\sigma}+e_{\sigma} \rightarrow \lambda\right)^{-} \text {and } U_{\text {odd }} M\left(\mu_{\sigma}+e_{\sigma} \rightarrow \lambda\right)^{-} \text {, }
$$

take a path $p=\left(\mu_{\sigma}+e_{\sigma}, n\right)$, being an element of $M\left(\mu_{\sigma}+\alpha_{\sigma} \rightarrow \lambda\right)^{-}$. Let $H_{j}$ be the last hyperplane of those hyperplanes in (2.2) the path meets. Then, by $(4.4), \Phi(p)$ is a path from $\mu_{\sigma(j, j+1}+\theta_{\sigma(j, j+1)}$ to $\lambda$ touching $H_{j}$, hence $\varphi(p)$ is an element of $M\left(\mu_{\sigma}(j, j+x)+\theta_{\sigma}(j, j+x) \rightarrow \lambda\right)^{-}$. Since is an involution, must be a bijection.

(6) Using (5.13) and (5.16), respectively, we obtain the following weight property of $\$$ :

$$
\text { maj } \$(p)=\text { maj }_{p}-\mu_{j}+\mu_{j+2}-1
$$

where $\mu$ is the initial point of $p$.

Finally, to illustrate action of $L_{\mathfrak{j}}, R_{\mathbf{j}}$ and $\$$, we give an example. Let $p_{1}=\left((1,-1,-1), m_{1}=31232223212131\right)$, which is a path from $\mu_{2}=(1,-1,-1)$ to $\lambda_{1}=(5,5,3)$. The function $i d_{1}\left(x_{1}, 1\right)$ reaches its minimal value at $\dot{F}=9$ and F=11, therefore by Definition $1(\mathrm{~A})(\mathrm{a}) \quad(\theta=6, f=7, \quad k=9, g=10, \quad r=8)$, $L_{1}\left(\pi_{1}\right)=31232221312131$, and by Definition $2(A)(c)(\theta=10, r=12, f=12, g=14$, k=14), $R_{1}\left(x_{1}\right)=31232223212321$. The function $i d_{2}\left(\pi_{1}, i\right)$ reaches its minimal value at $f=1$ and $f=4$, therefore by Definition $1(B)(c) \quad(e=0, k=1$, $f=1, \quad g=4, \quad r=3), \quad L_{2}\left(\pi_{1}\right)=12232223212131$, and by Definition $2(B)(b) \quad(\theta=1$, $f=4, \quad r=5, g=8, k=5), \quad R_{2}\left(x_{1}\right)=31233223212131$. We have maj $x_{1}=46$, $\operatorname{maj} L_{1}\left(\pi_{1}\right)=\operatorname{maj} L_{2}\left(\pi_{2}\right)=45$ in accordance with (5.13), and maj $R_{1}\left(\pi_{1}\right)=$ maj $R_{2}\left(x_{1}\right)=47$ in accordance with $(5.16)$. $H_{1}$ is the last 
hyperplane of $\left\{H_{1}, H_{2}\right\}$ p touches, $\mu_{1}-\mu_{2}+1=3$, therefore we obtain

$$
+\left(p_{1}\right)=\left(R_{1}(1,-1,-1), L_{2}^{3}\left(x_{1}\right)\right)=((-2,2,-1), 31232111312131) .
$$

Indeed this is a path from $(-2,2,-1)$ to $\lambda_{1}=(5,5,3)$ with maj $\left(p_{2}\right)=43=$ $=\operatorname{maj} p_{1}-3$ in accordance with (5.25).

\$ serves to evaluate $\mathcal{R}(\mu, \lambda ; q)^{+}$and $\bar{F}^{\prime}(\mu \rightarrow \lambda ; q)^{+}$, but we need another bijection with the help of which $\bar{F}(\mu \rightarrow \lambda ; q)^{+}$and $F^{\prime}(\mu \rightarrow \lambda ; q)^{+}$can be evaluated. We shall only give the analogous definitions in order to

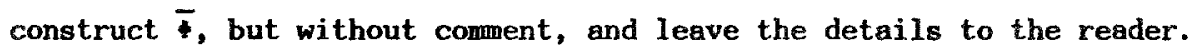

DEFINITION 4. With the assumptions of Definition 1 we define an alternative "lower" function $\overline{L_{\mathrm{j}}}$ by

(A) If $\pi$ satisfies

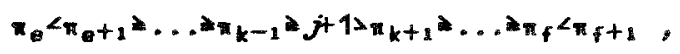

where $1 \leqslant e+1<f \leqslant p$, and

$$
\begin{array}{ll}
\text { either (a) } e+1<k<f \\
\text { or } & \text { (b) } k=+1 \text { and } \pi_{e}<\pi_{e+2} \\
\text { or } & \text { (c) } k=f \text { and } \pi_{f-1}<\pi_{f+1} \text {, }
\end{array}
$$

then

$\overline{L_{j}}(x)=\pi_{1} \ldots \pi_{e} \pi_{e+1} \ldots \pi_{k-1} \pi_{k+1} \ldots \pi_{r-1} j \pi_{r} \ldots \pi_{k} n_{f+1} \ldots \pi_{p}$

where $r$ is minimal with

$$
\vec{A}_{\pi_{r}} \text { and e+1 } \leqslant r \leqslant f \text {, }
$$

if there is none, $r=f+1$.

(B) If n satisfies

$$
\pi_{e} z_{k+1}<\ldots<\pi_{k-1}<j+1<\pi_{k+1}<\ldots<\pi_{f} \|_{\pi_{f+1}},
$$

where $1 \leqslant e+1<f^{k} p$, and

$$
\begin{array}{ll}
\text { either (a) } e+1<k<f \\
\text { or } & \text { (b) } k=e+1 \text { and } n_{f} n_{e+2} \\
\text { or } & \text { (c) } k=f \text { and } n_{f-2} n_{f+1},
\end{array}
$$

then

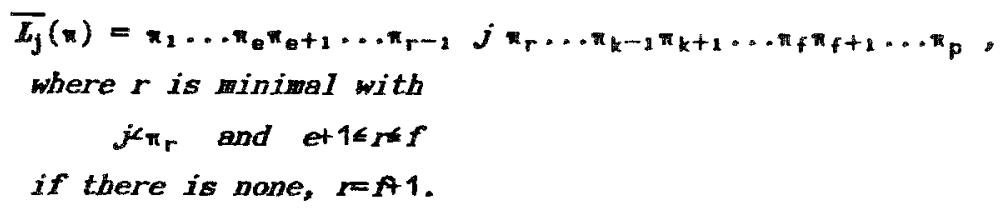


DEFINITION 5. With the assumptions of Definition 2 we define an alternative "raise" function $\boldsymbol{R}_{\mathrm{J}}$ by

(A) If n satisfies

$$
n_{e}<\pi_{\theta+2} \geqslant \ldots \pi_{r}-1>\lambda \pi_{r+1} \geqslant \ldots \geqslant n_{f}<n_{f+1}
$$

where $1 \leqslant \mathrm{e}+1<f \leq p$, and

$$
\begin{array}{ll}
\text { either (a) et } 1<p<f \\
\text { or } & \text { (b) } r=e+1 \text { and } n_{e}<n_{e+2} \\
\text { or } & \text { (c) } r=f \text { and } n_{f-1}<n_{f+1} \text {, }
\end{array}
$$

then

$$
\overline{R_{j}}(n)=n_{1} \ldots n_{e} n_{e+1} \ldots \pi_{k-1} j+1 n_{k} \ldots \pi_{r-1} n_{r}+1 \ldots n_{f} n_{f+1} \ldots n_{p} \text {, }
$$

where $k$ is minimal with

$$
j+1 x_{k} \text { and e+14kff. }
$$

if there is none, $l=f+1$.

(B) If * satisfies

$$
n_{e} m_{e+1}<\ldots<\pi_{r-1}<j<\pi_{r+1}<\ldots<n_{f} m_{n_{f+2}}
$$

where $1 \leqslant \mathrm{e}+1<f<p$, and

$$
\begin{array}{ll}
\text { either (a) } e+1<r<f \\
\text { or } & \text { (b) } r=e+1 \text { and } \pi_{e} m_{e+2} \\
\text { or } & \text { (c) } r=f \text { and } \pi_{f-1} m_{f+1},
\end{array}
$$

then

$\overline{B_{j}}(n)=n_{1} \ldots n_{e} n_{e+1} \ldots n_{r-1} n_{r+1} \ldots n_{k-1} j+1 n_{k} \ldots n_{f} n_{f+1} \ldots n_{p}$,

where $k$ is rinimal with

$$
j+1<u_{k} \text { and e+1<ktf, }
$$

if there is none, $l=f+1$.

DBFINITION 6. With the assumptions of Definition $3, \overline{4}$ is defined by

$$
\bar{\phi}(p)=\left(R_{\mathrm{j}}(\mu), \bar{L}_{\mathrm{j}} \mu_{\mathrm{j}}-\mu_{\mathrm{j}+1}+1(\pi)\right)
$$

where, if $\mu_{j}-\mu_{j+1}+1<0, \overline{L_{j}} \mu_{j}-\mu_{j+1}+1$ means $\overline{R_{j}}-\left(\mu_{j}-\mu_{j+1}+1\right)$.

What we need in the sequel is, that is a bijection between

$$
\underset{\sigma \operatorname{Uven}}{\mathrm{U}}\left(\mu_{\sigma}+e_{\sigma} \rightarrow \lambda\right)^{-} \text {and } \mathrm{Jdd}_{\mathrm{odd}}^{\mathrm{U}} M\left(\mu_{\sigma}+e_{\sigma} \rightarrow \lambda\right)^{-}
$$

and the validity of the weight property

$$
\overline{\mathbf{m a j}} \overline{(p)}=\overline{\overline{\mathrm{maj}}} \mathbf{p}
$$




\section{LATTICE PATH BNUMBRATION.}

THEOREM 1. Let $\mu . \lambda \varepsilon D_{n}$ and $\lambda \geq \mu$. Then

(6.1) $\quad F(\mu \rightarrow \lambda ; q)^{+}=[|\lambda-\mu|] ! \operatorname{det}\left(q^{\left(\mu_{t}+s-t\right)-\left(\begin{array}{c}\mu_{t} \\ 2\end{array}\right)} /\left[\lambda_{s}-s-\mu_{t}+t\right] !\right)$.

PROOF. We introduce a weight function $w_{1}$, acting on elements of of $\mathrm{U}_{n}\left(\mu_{\sigma}+e_{\sigma} \rightarrow \lambda\right)^{-}$. Let $p=\left(\mu_{\sigma}+\varepsilon_{\sigma}, \pi\right)$ be an element of $M\left(\mu_{\sigma}+\varepsilon_{\sigma} \rightarrow \lambda\right)^{-}$for

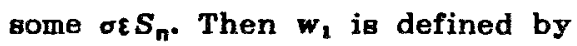

$$
\omega_{1}(p)=q^{\sum^{p}}\left(\begin{array}{c}
{\left[\mu_{\sigma}+e_{d}\right]_{t}} \\
2
\end{array}\right) \cdot q^{\operatorname{maj} p} .
$$

Let $H_{\mathrm{f}}$ be the last hyperplane of (2.2) meets. By (5.18) and (5.25)

$$
w_{1}(t(p))=q^{K} \cdot q^{m a j} p-\left[\mu_{\sigma}+e_{\sigma}\right]_{j}+\left[\mu_{\alpha}+e_{\sigma}\right]_{j+1}-1
$$

where

$$
K=\sum_{t=1}^{n}\left(\begin{array}{c}
{\left[R_{j}\left(\mu_{\sigma_{1}}+e_{\sigma}\right)\right]_{t}} \\
2
\end{array}\right]
$$

Since, regarding (4.4), $\left[R_{j}\left(\mu_{\sigma}+e_{\sigma}\right)\right]_{j}=\mu_{\sigma}(j+1)+j-\sigma(j+1) \quad$ and $\left[R_{j}\left(\mu_{\sigma}+\theta_{\sigma}\right)\right]_{j+1}=\mu_{\sigma}(j)+j+1-\sigma(j)$, we obtain

$$
\begin{aligned}
& {\left[\frac{\left[R_{j}\left(\mu_{\sigma}+\varepsilon_{\sigma}\right)\right]_{j}}{2}\right)+\left(\frac{\left[R_{j}\left(\mu_{\sigma}+\varepsilon_{\sigma}\right)\right]_{j+2}}{2}\right)=} \\
& =\left(\begin{array}{c}
\mu_{\sigma}(j+1)+j+1-\sigma(j+1) \\
2
\end{array}\right]-\left(\mu_{\sigma(j+1)}+j \sigma(j+1)\right)+ \\
& +\left(\mu_{\sigma}(j)+j \sigma(j)\right)+\left(\mu_{\sigma}(j)+j-\sigma(j)\right) \\
& =\left[\begin{array}{c}
{\left[\mu_{\sigma}+\varepsilon_{\sigma}\right]_{j+1}} \\
2
\end{array}\right]+\left[\begin{array}{c}
{\left[\mu_{\sigma}+\varepsilon_{\sigma}\right]_{j}} \\
2
\end{array}\right]-\left[\mu_{\sigma}+\varepsilon_{\sigma}\right]_{j+1}+1+\left[\mu_{\sigma}+\varepsilon_{\sigma}\right]_{j} .
\end{aligned}
$$

Therefore (6.3) becomes

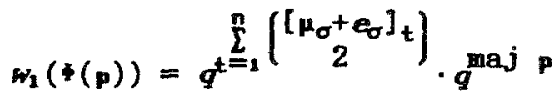

$$
\begin{aligned}
& =\omega_{1}(p) \text {. }
\end{aligned}
$$

which says that is weight-preserving with respect to $w_{1}$. Therefore, having in mind Remark (5) after Definition 3, the equation which replaces (4.2) reads 
KRATTENTHALER

$$
\begin{aligned}
\sigma \sum_{\text {even }} g^{\sum_{t=1}^{n}\left[\begin{array}{c}
{\left[\mu_{\sigma}+e_{\sigma}\right]_{t}} \\
2
\end{array}\right]} \underset{F\left(\mu_{\sigma}+e_{\sigma} \rightarrow \lambda ; q\right)^{-}}{ }= \\
=\sigma \sum_{\sigma d d} q^{\sum_{=1}^{n}}\left[\begin{array}{c}
{\left[\mu_{\sigma}+e_{\sigma}\right]_{t}} \\
2
\end{array}\right] \quad F\left(\mu_{\sigma}+e_{\sigma} \rightarrow \lambda ; q\right)^{-} .
\end{aligned}
$$

The identity which replaces (4.1) is MacMahon's well-known result [1, Theorem 3.7, p.42], which in our notation is

$$
\Gamma(\mu \rightarrow \lambda ; g)=\left[\begin{array}{c}
|\lambda-\mu| \\
\lambda-\mu
\end{array}\right] \text {. }
$$

Successive use of (6.4). (4.5), and (6.5) gives

$$
\begin{aligned}
& q^{\sum_{t=1}^{n}\left(\mu_{t}\right)} F(\mu \lambda ; q)^{+}= \\
& =g^{\sum^{\sum_{=1}^{n}}\left(\begin{array}{c}
\mu_{t} \\
2
\end{array}\right)} F(\mu \rightarrow \lambda ; q)-g^{\sum_{=1}^{n}\left(\mu_{2}^{\mu_{t}}\right)} F(\mu \rightarrow \lambda ; q)^{-}
\end{aligned}
$$

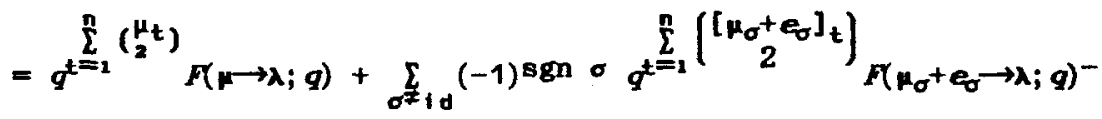

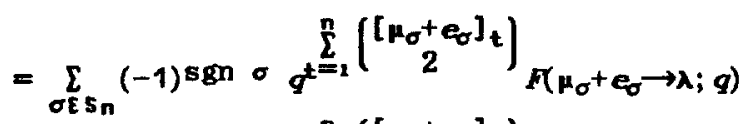

$$
\begin{aligned}
& =\sum_{\sigma \varepsilon S_{n}}(-1)^{\operatorname{sgn} \sigma} g^{\sum^{t=2}}\left(\begin{array}{c}
{\left[\mu_{\sigma}+e_{\sigma}\right] t} \\
2
\end{array}\right)\left[\begin{array}{c}
|\lambda-\mu| \\
\lambda-\mu_{\sigma}-\varepsilon_{\sigma}
\end{array}\right] \\
& =[|\lambda-\mu|] ! \operatorname{det}\left(q^{\left(\mu_{t}+s-t\right)} /\left[\lambda_{s}-s-\mu_{t}+t\right] !\right) \text {, }
\end{aligned}
$$

which after division of $g^{\left(\mu_{2}^{\mu_{t}}\right)}$ turns into (6.1). D

THBOREM 2. Let $\mu, \lambda \in D_{n}$ and $\lambda \geq \mu$. Then

(6.6) $\bar{F}^{\prime}(\mu \rightarrow \lambda ; q)^{+}=[|\lambda-\mu|] ! \operatorname{det}\left(q^{\left(-\lambda_{s}+s-\phi\right.}-\left(\begin{array}{c}-\lambda_{s} \\ 2\end{array}\right) /\left[\lambda_{s}-s-\mu_{t}+t\right] !\right)$.

SKETCH OF PROOF. Define a bijection on $\mathrm{Z}^{\mathrm{n}}$ by

$$
\left(x_{1}, \ldots, x_{1}, \ldots, x_{n}\right) \longrightarrow\left(-x_{n}, \ldots,-x_{n-1+1}, \ldots,-x_{1}\right) .
$$

Extending to lattice paths $p$ by

$$
Y(p)=(p(p(1)), \ldots, t(p(p)))
$$

it is not difficult to verify

$$
\overline{\text { maj' }} p=\text { maj }(p)
$$

and

150 


$$
\left.(M \mu \rightarrow \lambda)^{+}\right)=M(\lambda(\lambda) \rightarrow(\mu))^{+}
$$

This furnishes

$$
\bar{F}^{\prime}(\mu \rightarrow \lambda ; q)^{+}=F(\boldsymbol{H}(\lambda) \rightarrow(\mu) ; q)^{+}
$$

Then short evaluation turns formula $(6.1)$, for $\mu$ replaced by $(\lambda)$ and $\lambda$ replaced by $(\mu)$, into $(6.5)$. a

THEOREM 3. Let $\mu, \lambda \in D_{n}$ and $\lambda i_{\mu}$. Then

$$
\bar{\mu}(\mu \rightarrow \lambda ; g)^{+}=[|\lambda-\mu|] ! \operatorname{det}\left(1 /\left[\lambda_{s}-s-\mu_{t}+t\right] !\right) .
$$

SKETCE OF PROOR. The proof runs through quite analogously like

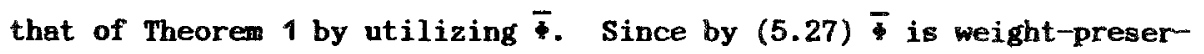
ving with respect to $\overline{\mathrm{maj}}$, we do not have to introduce an auxiliary weight (which in the proof of Theorem 1 was $w_{1}$ ). All other steps may be transferred almost verbatim.

THEOREM 4. Let $\mu, \lambda \varepsilon D_{n}$ and $\lambda \geq \mu$. Then

$$
F^{\prime}(\mu \rightarrow \lambda ; q)^{+}=[|\lambda-\mu|] ! \operatorname{det}\left(1 /\left[\lambda_{s}-\Sigma-\mu_{t}+t\right] !\right)
$$

SKBTCH OF PROOF. The map introduced in the proof of Theorem 2 Batisfies (6.7) and

$$
\mathbf{m a j} \mathbf{p}=\overline{\mathbf{m a j}}+(p)
$$

for all lattice paths p. This implies

$$
F^{\prime}(\mu \rightarrow \lambda ; q)^{+}=\bar{F}(\boldsymbol{\Psi}(\lambda) \rightarrow(\mu) ; q)^{+}
$$

which after short evaluation may be turned into (6.9), a

RFMARK. Of course there is no difficulty to give reflection-like proofs for Theorems 2 and 4 in the sense of Theorems 1 and 3 by introducing two further involutions, $\overline{\text { ' and }}$ ', with suitable weightproperties for $\overline{\mathbf{m a j}}$ and maj', respectively. We have avoided this because there do not arise new aspects, but application of the map makes the proofs of Theorems 2 and 4 shorter. The reader might try to find such involutions " and $\$$ '.

In the special case $\mu=0$ the determinants in Theorems $2-4$ may bo 
simplified. To this end we use the following lemma, which generalizes Vandermonde's determinant.

LBMMA 5. Let $x_{1}, x_{2}, \ldots, x_{n}, a_{2}, \ldots, a_{n}$ be indeterminates. Then

$$
\operatorname{det}\left(\left(x_{8}+a_{n}\right)\left(x_{8}+a_{n-1}\right) \cdots\left(x_{8}+a_{t+1}\right)\right)=\prod_{k_{t}}\left(x_{8}-x_{t}\right),
$$

if, by convention, for $t=n$ the entries in the $n \times n$ determinant are set equal to 1.

PROOF. The determinantal expression on the left-hand side of (6.10) is a polynomial in $x_{1}, x_{2}, \ldots, x_{n}$ with degree $\left(\frac{n}{2}\right)$. If for $s_{1}, s_{2}$ with $1 \leqslant s_{1}<s_{2} \leqslant D$ we have $x_{s_{1}}=x_{s_{2}}$, the $s_{1}$-th and $s_{2}$-th row in the determinant are identical. Therefore the determinant may be factorized

(6. 11) $\operatorname{det}\left(\left(x_{8}+a_{n}\right)\left(x_{8}+a_{n-1}\right) \cdots\left(x_{s}+a_{t+2}\right)\right)=\prod_{g}\left(x_{t}-x_{t}\right) \cdot p\left(x_{1}, \ldots, x_{n}\right)$, where $p\left(x_{1}, \ldots, x_{n}\right)$ is some polynomial in $x_{1}, \ldots, x_{n}$. But the degree of $\prod_{g<t}\left(x_{g}-x_{t}\right)$ is $\left(\begin{array}{l}n \\ 2\end{array}\right)$, therefore $p\left(x_{1}, \ldots, x_{n}\right)$ is identical to a constant. Considering the product of the main-diagonal, it develops that the coefficient of the monomial $x_{1}^{n-1} x_{2}^{n-2} \cdots x_{n-1} x_{n}^{0}$ on the left-hand side of (6.11) must be equal to 1 , hence $p\left(x_{1}, \ldots, x_{n}\right)=1$. $\quad$

COROLLARY 6. Let $\lambda E D_{n}, \lambda \geq 0$ and $|\lambda|=p$. Then

$$
\bar{F}^{\prime}(0 \rightarrow \lambda ; q)^{+}=\frac{[p] !}{\left[d_{1}\right] \cdots\left[d_{p}\right]}
$$

and

$$
\bar{F}(0 \rightarrow \lambda ; q)^{+}=F^{\prime}(0 \rightarrow \lambda ; q)^{+}=q^{\mathscr{L}_{1}} \frac{[p] !}{\left[d_{1}\right] \cdots\left[d_{p}\right]},
$$

where $K_{1}=\sum_{i=1}^{n}(i-1) \lambda_{1}$, and $d_{1}, \ldots, d_{p}$ denote the hook-length of $\lambda$ (see [11, Definition 15.1]).

ProOF. Setting $p=0$ in (6.6) gives

$$
\begin{aligned}
& \bar{F}^{\prime}(0 \rightarrow \lambda ; q)^{+}=[|\lambda|] ! \operatorname{det}\left(g^{\left[\begin{array}{l}
-\lambda_{s}+s-t \\
2
\end{array}-\left(\begin{array}{c}
-\lambda_{2} \\
2
\end{array}\right]\right.} /\left[\lambda_{s}-s+t\right] !\right)
\end{aligned}
$$

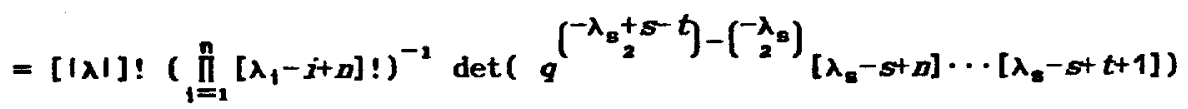

$$
\begin{aligned}
& =[|\lambda|] !\left(\prod_{i=1}^{n}\left[\lambda_{1}-i+n\right] !\right)^{-1} q^{K_{2}} \text {. } \\
& \cdot \operatorname{det}\left(\left(g^{-\lambda_{s}+s}\left[\lambda_{s}-s\right]+[n]\right) \cdots\left(q^{-\lambda_{s}+s}\left[\lambda_{s}-s\right]+[t+1]\right)\right) \text {, }
\end{aligned}
$$


where $K_{2}=\sum_{i=1}^{n}\left(\lambda_{1}(n-i)+i^{2}-n i\right)$. Application of Lemma 5 with $x_{3}=$ $=q^{-\lambda_{s}+s}\left[\lambda_{B}-s\right]$ and $a_{i}=[i]$ yields

$$
\begin{aligned}
& \bar{F}^{\prime}(0 \rightarrow \lambda ; q)^{+}=[|\lambda|]:\left(\prod_{1=2}\left[\lambda_{1}-i+D\right] !\right)^{-\varepsilon} q^{K_{2}} . \\
& \cdot \prod_{g<t}\left(g^{-\lambda_{s}+s}\left[\lambda_{s}-s\right]-g^{-\lambda_{t}+t}\left[\lambda_{t}-t\right]\right) \\
& =[|\lambda|] !\left(\prod_{i=1}^{n}\left[\lambda_{1}-i+n\right] !\right)^{-2} g^{K_{3}} \prod_{g}\left[\lambda_{8}-\theta-\lambda_{t}+t\right] .
\end{aligned}
$$

where

$$
A_{3}=\sum_{i=1}^{n}\left(\lambda_{i}(n-i)+i^{2}-n i\right)+\sum_{i=1}^{n}\left(-\lambda_{i}+i\right)(n-i)=0
$$

The proof of (6.12) is completed by the observation

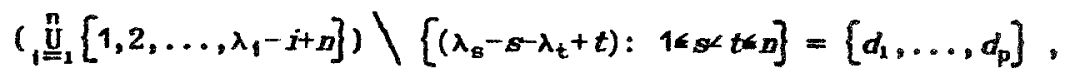

when being regarded as a multiset identity. (See for example [7, g.9, identity (4) in Example 11.)

In order to prove (6.13) set $=0$ in (6.8) and (6.9), respectively, getting

$$
\begin{aligned}
\bar{F}(0 \rightarrow \lambda ; q)^{+}= & F^{\prime}(0 \rightarrow \lambda ; q)^{+}= \\
= & {[|\lambda|] !\left(\prod_{i=1}^{n}\left[\lambda_{i}-i+n\right] !\right)^{-1} \operatorname{det}\left(\left[\lambda_{s}-s+n\right] \cdots\left[\lambda_{s}-s+t+1\right]\right) } \\
= & {[|\lambda|] !\left(\prod_{i=1}^{n}\left[\lambda_{1}-i+n\right] !\right)^{-1} g^{h_{4}} . } \\
& \cdot \operatorname{det}\left(\left(\left[\lambda_{s}-s\right]+q^{-n}[n]\right) \cdots\left(\left[\lambda_{s}-s\right]+q^{-t-1}[t+1]\right)\right),
\end{aligned}
$$

where

$$
K_{4}=\sum_{i=1}^{n}\left(\left({ }_{2}^{n+1}\right)-\left(i_{2}^{+1}\right)\right]=n\left(\begin{array}{c}
n+1 \\
2
\end{array}\right)-\left(\begin{array}{c}
n+2 \\
3
\end{array}\right)=2\left(\begin{array}{c}
n+1 \\
3
\end{array}\right)
$$

Application of Lemma 5 with $x_{\varepsilon}=\left[\lambda_{s}-8\right]$ and $a_{i}=q^{-i}[i]$ leads to

$$
\begin{aligned}
\bar{F}(0 \rightarrow \lambda ; q)^{+} & =F^{\prime}(0 \rightarrow \lambda ; q)^{+}= \\
& =[|\lambda|] !\left(\prod_{1=1}^{n}\left[\lambda_{1}-i+n\right] !\right)^{-\lambda} q^{K_{5}} \prod_{\alpha_{t}}\left[\lambda_{s}-s-\lambda_{t}+t\right],
\end{aligned}
$$

where

$$
\kappa_{5}=2\left(n_{3}^{1}\right)+\sum_{i=1}^{n}\left(\lambda_{i}-i\right)(i-1)=\sum_{i=1}^{n}(i-1) \lambda_{i} \cdot 0
$$

It is an old open problem to simplify the determinant in (6.1) for $\mu=0$. Unfortunately, Lemma 5 , used above to establish Corollary 6 , seemingly 
does not apply to this case.

\section{GENERATING FUNCTIONS FOR SKEW PLANE PARTITIONS OF A GIVEN}

SHAPE. As corollaries of Theorems 1 to 4 we obtain expressions for the generating functions of the various kinds of skew plane partitions.

COROLLARY 7. The generating function for skew plane partitions of the given shape $\lambda / \mu$ is

$$
g^{|\lambda-\mu|} \operatorname{det}\left(q^{\left(\mu_{t}+s-t\right)-\left(\frac{\mu_{2}}{2}\right)} /\left[\lambda_{B}-s-\mu_{t}+t\right] !\right) .
$$

ProOF. We only have to combine (3.8), (3.9), and (6.1). 口

COROLLARY 8. The generating function for reverse skew plane partitions of shape $\lambda / \mu$ is

$$
q^{|\lambda-\mu|} \operatorname{det}\left(q^{\left(\begin{array}{l}
\lambda_{\mathrm{g}}+s-t \\
2
\end{array}-\left(\begin{array}{c}
-\lambda_{2} \\
2
\end{array}\right)\right.} /\left[\lambda_{\mathrm{s}}-s-\mu_{t}+t\right] !\right)
$$

The generating function for reverse plane partitions of shape $\lambda$ is

$$
q^{p} /\left[d_{1}\right] \cdots\left[d_{p}\right]
$$

where $|\lambda|=p$.

PROOF. For (7.2) combine (3.12), (3.13), and (6.6). (7.3) arises when combining (3.12), (3.13) for $\mu=0$, and (6.12). $\quad$

Considering (3.10), (3.11), (6.9); and (3.14), (3.15), (6.8); and (6.13); respectively, we obtain

COROLLARY 9. The generating function for column-gtrict skew plane partitions or column-strict reverse skew plane partitions, respectively, of shape $\lambda / \mu$ is

$$
q^{|\lambda-\mu|} \operatorname{det}\left(1 /\left[\lambda_{s}-s-\mu_{t}+t\right] !\right)
$$

The generating function for column-strict plane partitions or column-strict reverse plane partitions, respectively, of shape $\lambda$ is

$$
q^{L} /\left[d_{1}\right] \cdots\left[d_{p}\right],
$$

where $|\lambda|=p$ and $F=\sum_{i=1}^{n} i \lambda_{1} \cdot 0$ 
1. ANDREWS,G.E.: The theory of partitions, Reading, Masaachusetis: Addison-Wesley 1976

2. FRANZBLAU,D.S., ZEILBERGER,D.: A bijective proof of the hook-length formula, J. Algorithmus 3,3 17-343(1982)

3. FURLINGER,J., HOFBAUER,J.: q-Catalan numbers, J. Combin. Theory A 40, 248-264(1985)

4. GANSNER,E.: The Hillman-Grasal correspondence and the enumeration of reverse plane partitions, J. Combin. Theory A 30, 71-89(1981)

5. GESSEL,I., VIENNOT,G.: Binomial determinants, paths, and hook-length formulae, Adv. in Math. 58, 300-321(1985)

6. HILLMAN,A.P., GRASSL,R.M.: Reverse plane partitions and tableaux hook numbers, J. Combin. Theory A 21, 216-221(1976)

7. MACDONALD,I.G.: Symmetric functions and Hall polynomials, Oxford: Clarendon Press 1979

8. MACMAHON,P.A.: Combinatory analysis, Vol.2, London: Cambridge University Press 1915-1916; reprint Chelsea-New York 1960

9. REMMEL,J.B., WHITNEY,R.: A bijective proof of the generating function for the number of reverse plane partitions via latice paths, Linear and Multilinear Algebra 16, 75-91(1984)

10. STANLEY,R.P.: Ordered structures and partitions, Mem. Amer. Math. Soc. 119(1972)

11. STANLEY,R.P.: Theory and applications of plane partitions, Part 1: Studies Appl. Math. 50, 167-188(1971); Part 2: Studies Appl. Math. 50, 259-279(1971)

12. WATANABE,T., MOHANTY,S.G.: On an inclusion-exclusion formula based on the reflection principle, Discrete Math. 64, 281-288(1987)

13. ZEILBERGER,D.: Andre's reflection proof generalized to the manycandidate ballot problem, Discrete Math. 44, 325-326(1983)

Institut für Mathematik

Universität Wien

Strudlhofgasse 4

A-1090 Vienna

AUSTRIA 